\title{
New ultra metal-poor stars from SDSS: follow-up GTC medium-resolution spectroscopy ${ }^{\star}$
}

\author{
D. S. Aguado ${ }^{1,2}$, C. Allende Prieto ${ }^{1,2}$, J. I. González Hernández ${ }^{1,2}$, R. Rebolo ${ }^{1,2,3}$, and E. Caffau ${ }^{4}$ \\ ${ }^{1}$ Instituto de Astrofísica de Canarias, vía Láctea, 38205 La Laguna, Tenerife, Spain \\ e-mail: aguado@iac.es \\ 2 Universidad de La Laguna, Departamento de Astrofísica, 38206 La Laguna, Tenerife, Spain \\ 3 Consejo Superior de Investigaciones Científicas, 28006 Madrid, Spain \\ ${ }^{4}$ GEPI, Observatoire de Paris, PSL Research Univsersity, CNRS, place Jules Janssen, 92190 Meudon, France
}

Received 6 June 2017 / Accepted 13 June 2017

\begin{abstract}
Context. The first generation of stars formed in the Galaxy left behind the chemical signatures of their nucleosynthesis in the interstellar medium, visible today in the atmospheres of low-mass stars that formed afterwards. Sampling the chemistry of those low-mass provides insight into the first stars.

Aims. We aim to increase the samples of stars with extremely low metal abundances, identifying ultra metal-poor stars from spectra with modest spectral resolution and signal-to-noise ratio $(\mathrm{S} / \mathrm{N})$. Achieving this goal involves deriving reliable metallicities and carbon abundances from such spectra.

Methods. We carry out follow-up observations of faint, $V>19$, metal-poor candidates selected from SDSS spectroscopy and observed with the Optical System for Imaging and low-Intermediate-Resolution Integrated Spectroscopy (OSIRIS) at GTC. The SDSS and follow-up OSIRIS spectra were analyzed using the FERRE code to derive effective temperatures, surface gravities, metallicities and carbon abundances. In addition, a well-known extremely metal-poor star has been included in our sample to calibrate the analysis methodology.

Results. We observed and analyzed five metal-poor candidates from modest-quality SDSS spectra. All stars in our sample have been confirmed as extremely metal-poor stars, in the $[\mathrm{Fe} / \mathrm{H}]<-3.3$ regime. We report the recognition of J173403+644632, a carbonenhanced ultra metal-poor dwarf star with $[\mathrm{Fe} / \mathrm{H}]=-4.3$ and $[\mathrm{C} / \mathrm{Fe}]=+3.1$.
\end{abstract}

Key words. stars: abundances - stars: Population II - Galaxy: abundances - galaxies: formation - galaxies: halos

\section{Introduction}

Metal-poor stars are extremely rare objects. Their study is key to understanding the early Galaxy. The fraction of stars with low metal content increases with magnitude (Robin et al. 2003) and increases at high Galactic latitudes. In addition, the number of halo stars at $[\mathrm{Fe} / \mathrm{H}] \sim-4$ is only a small percent of those at $[\mathrm{Fe} / \mathrm{H}] \sim-3$, and those are only a small percent of those at $[\mathrm{Fe} / \mathrm{H}] \sim-2$ (Allende Prieto et al. 2016). About two dozen stars are known at $[\mathrm{Fe} / \mathrm{H}]<-4$ (Bonifacio et al. 2015; Placco et al. 2015; Aguado et al. 2016) even though it is the most interesting regime on the yields from the very first generation of stars. Developing new procedures to identify faint metal-poor stars is an important task.

We have been trying to identify these rare objects from large ongoing spectroscopy surveys (SDSS, LAMOST, etc.), to reobserve them at medium-resolution, and to derive reliable stellar atmospheric parameters, including metallicity and whenever possible carbon abundances (Aguado et al. 2016, 2017). This method can be used to identify extremely metal-poor stars as faint as $V \sim 19$. Before performing high-resolution spectroscopy to derive multiple chemical abundances, having an intermediate medium-resolution step provides a powerful time-saving tool

\footnotetext{
* Based on observations made with the Gran Telescopio Canarias (GTC), installed in the Spanish Observatorio del Roque de los Muchachos of the Instituto de Astrofísica de Canarias, on the island of La Palma. Programme ID GTC2E-16A and ID GTC65-16B.
}

with regard to large telescopes (6-10 m class) since it dramatically removes false positives in the analysis of large-scale spectroscopic surveys. With this methodology, a large sample of ultra-metal poor stars can be assembled in preparation for the arrival of the next $30 \mathrm{~m}$ class generation of telescopes.

This is the third paper in a series devoted to follow-up spectroscopy of extremely and ultra metal-poor stars identified from SDSS and other large-scale spectroscopic surveys. In this work we report on faint candidates observed with the Optical System for Imaging and low-Intermediate-Resolution Integrated Spectroscopy (OSIRIS) at Gran Telescopio de Canarias (GTC). We also report on the recognition of $\mathrm{J} 173403+644632$, which at $g_{\text {mag }}=19.6$ is the faintest carbon-enhanced ultra metal-poor dwarf star known. The target selection is explained in Sect. 2, which provides a full description of the procedure originally described in Aguado et al. (2016). The observations and data reduction are detailed in Sect. 3. The derived atmospheric parameters are analyzed and discussed in Sect. 4. In addition, a study of the most metal-poor star known, J1029+1729, is developed to test our methodology in Sect. 4.1. Conclusions are given in Sect. 5.

\section{SDSS analysis and target selection}

We have re-analyzed more than a million spectra ( $90 \%$ stars) from the original Sloan Digital Sky Survey (York et al. 2000), the Sloan Extension for Galactic Understanding and Exploration 
Table 1. Coordinates and atmospheric parameters for the program stars based in the analysis of the low-resolution spectra with the FERRE code.

\begin{tabular}{|c|c|c|c|c|c|c|c|c|}
\hline Star & $\begin{array}{c}g \\
\text { mag }\end{array}$ & $\begin{array}{c}\text { RA } \\
h^{\prime \prime \prime \prime}\end{array}$ & $\begin{array}{l}\text { Dec } \\
\circ, " 1,\end{array}$ & $\begin{array}{c}T_{\text {eff }} \\
\mathrm{K}\end{array}$ & $\begin{array}{c}\log g \\
\mathrm{~cm} \mathrm{~s}^{-2}\end{array}$ & {$[\mathrm{Fe} / \mathrm{H}]$} & {$[\mathrm{C} / \mathrm{Fe}]$} & $\langle S / N\rangle^{a}$ \\
\hline SDSS J012512+070319 & 19.0 & $01: 25: 12.5$ & $+07: 03: 19.8$ & 6454 & 4.9 & -3.2 & 0.8 & 22 \\
\hline SDSS J015131+163944 & 18.9 & $01: 51: 31.2$ & $+16: 39: 44.9$ & 5917 & 3.9 & -3.6 & 1.3 & 19 \\
\hline SDSS J041800+062308 & 19.1 & 04:18:00.7 & $+06: 23: 08.1$ & 5579 & 0.5 & -4.3 & - & 21 \\
\hline SDSS J094708+461010 & 19.1 & 09:47:08.2 & $+46: 10: 10.1$ & 5916 & 4.9 & -4.7 & 0.2 & 14 \\
\hline SDSS J173403+644632 & 19.6 & $17: 34: 03.9$ & $+64: 46: 32.9$ & 6470 & 4.9 & -3.8 & -0.2 & 15 \\
\hline Star & Plate & mjd & Fiberid & $\begin{array}{c}T_{\mathrm{SSPP}} \\
\mathrm{K}\end{array}$ & $\begin{array}{c}\log g_{\mathrm{SSPP}} \\
\mathrm{cm} \mathrm{s}^{-2}\end{array}$ & {$[\mathrm{Fe} / \mathrm{H}]_{\mathrm{SSPP}}$} & & \\
\hline SDSS J012512+070319 & 4556 & 55912 & 180 & - & - & - & & \\
\hline SDSS J015131+163944 & 5118 & 55830 & 336 & - & - & - & & \\
\hline SDSS J041800+062308 & 2826 & 54389 & 171 & 6387 & 2.70 & -3.26 & & \\
\hline SDSS J094708+461010 & 4695 & 55957 & 944 & - & - & - & & \\
\hline SDSS J173403+644632 & 2561 & 54597 & 501 & 6229 & 3.11 & -3.02 & & \\
\hline
\end{tabular}

Notes. The SDSS identification and parameters derived by the automatic SSPP are listed below (when available). ${ }^{(a)}$ Signal-no-noise ratios have been calculated as the average value for the entire SDSS spectrum.

(SEGUE, Yanny et al. 2009) and the Baryonic Oscillations Spectroscopic Survey (BOSS, Eisenstein et al. 2011; Dawson et al. 2013). The main stellar parameters (effective temperature $T_{\text {eff }}$, surface gravity $\log g$, and metallicity $\left.[\mathrm{Fe} / \mathrm{H}]^{1}\right)$ and the carbon abundance have been derived.

The SDSS optical spectra are from SDSS Data Release 9 (DR9, Ahn et al. 2012) for observations with the original SDSS spectrograph, and DR12 (Alam et al. 2015) for data obtained with the upgraded BOSS spectrographs (Smee et al. 2013). The resolving power of SDSS spectra is about 2000, covering the range $\sim 3800-9100 \AA$. Since the integration time is the same for all the targets, the signal-to-noise ratio $(\mathrm{S} / \mathrm{N})$ is widely variable. We use the fortran FERRE code (Allende Prieto et al. 2006) to perform a massive analysis of the spectra, as explained in Aguado et al. (2016 and 2017). For this work we focus on promising candidates with low $\mathrm{S} / \mathrm{N}$ spectra and, consequently, higher error bars in the parameter determination (see Table 1). These targets are too faint for follow-up with the $4.2 \mathrm{~m}$ WHT, and so a parallel program was started on the $10.4 \mathrm{~m} \mathrm{GTC}$.

\section{Observations and data reduction}

We performed medium-resolution, long-slit spectroscopy with OSIRIS at the $10.4 \mathrm{~m}$ telescope GTC. The program was granted $28 \mathrm{~h}$ under proposals ID GTC2E-16A and ID GTC65-16B in service mode to carry out long-slit spectroscopy with the R2500U grism and a 1.0 arcsec slit, providing a spectral range 3600 $4500 \AA$ with a resolving power $(R \equiv \lambda / \delta \lambda \sim 2300)$. The slit was in all cases oriented at the paralactic angle. The individual exposures were never longer than $30 \mathrm{~min}$ to minimize the impact of cosmic rays (see Table 2).

Data reduction consisted in bias substraction, flat-fielding, wavelength calibration using $\mathrm{HgAr}+\mathrm{Xe}$ calibration lamps, and a combination of individual spectra. The processing was performed with the twodspec and onespec packages in $\mathrm{IRAF}^{2}$

\footnotetext{
1 We use the bracket notation to report chemical abundances: $[a / b]=$ $\log \left(\frac{N(a)}{N(b)}\right)-\log \left(\frac{N(a)}{N(b)}\right)_{\odot}$, where $N(x)$ represents number density of nuclei of element $x$.

2 IRAF is distributed by the National Optical Astronomy Observatory, which is operated by the Association of Universities for Research in Astronomy (AURA) under cooperative agreement with the National Science Foundation.
}

Table 2. Observing log.

\begin{tabular}{ccccc}
\hline \hline Stars & Date & $N_{\exp }$ & $\begin{array}{c}t_{\exp } \\
\mathrm{s}\end{array}$ & Seeing \\
\hline J012512+070319 & 27-Sep.-2016 & 6 & 1600 & 0.8 \\
J015131+163944 & 22/28-Sep.-2016 & 7 & 1600 & 1.1 \\
J041800+062308 & 26/28-Sep.-2016 & 6 & 1600 & 1.0 \\
J094708+461010 & 28/31-Mar.-2016 & 10 & 1827 & 0.9 \\
J173403+644632 & 29/30-Apr.-2016 & 10 & 1827 & 1.0 \\
\hline G64-12 & 1-Jan.-2017 & 3 & 30 & 1.2 \\
\hline
\end{tabular}

Notes. A slit width of 1 arcsec. The moon was gray during all the observations and clouds were not noticeable.

(Tody 1993). Cross-correlation between individual spectra and correction of radial velocity offsets was made with the $r v$ package.

\section{Analysis and discussion}

Following the same procedure described by Aguado et al. (2017), we employed a grid of synthetic spectra computed with the ASS $\epsilon$ T code (Koesterke et al. 2008). The model atmospheres were computed as in Mészáros et al. (2012). The spectral synthesis was performed as described by Allende Prieto et al. (2014), but the grid we used differs in the parameter coverage: $-6 \leq$ $[\mathrm{Fe} / \mathrm{H}] \leq-2 ;+1 \leq[\mathrm{C} / \mathrm{Fe}] \leq+5 ; 4750 \mathrm{~K} \leq \mathrm{T}_{\text {eff }} \leq 7000 \mathrm{~K}$, and $1.0 \leq \log g \leq 5.0$. The $\alpha$-element abundance and microturbulence were fixed at $[\alpha / \mathrm{Fe}]=0.4$ and $2 \mathrm{~km} \mathrm{~s}^{-1}$, respectively. Lacking a reliable flux calibration for our observations, a running-mean filter with a window of 30 nearby pixels was used for continuum normalization. The same algorithm was applied to both observed and synthetic spectra after resampling the latter to the wavelength OSIRIS spectra of the former. Further details are given in Aguado et al. (2017). FERRE is used to simultaneously derive the three main stellar parameters - effective temperature, surface gravity, metallicity - as well as the carbon abundance.

Table 3 summarizes the results of the FERRE analysis for the sample stars. A high-quality OSIRIS spectrum of the wellknown metal-poor star G64-12 was analyzed as a reference. The good agreement between the optimal set of parameters 
Table 3. The stellar parameters and main results obtained from OSIRIS spectra.

\begin{tabular}{lccccccccc}
\hline \hline Star & $\langle S / N\rangle$ & $\begin{array}{c}T_{\text {eff }} \\
{[\mathrm{K}]}\end{array}$ & $\begin{array}{c}\Delta T_{\text {eff }} \\
{[\mathrm{K}]}\end{array}$ & $\begin{array}{c}\log g \\
{\left[\mathrm{~cm} \mathrm{~s}^{-2}\right]}\end{array}$ & $\begin{array}{c}\Delta \log g \\
{\left[\mathrm{~cm} \mathrm{~s}^{-2}\right]}\end{array}$ & {$[\mathrm{Fe} / \mathrm{H}]$} & $\Delta[\mathrm{Fe} / \mathrm{H}]$ & {$[\mathrm{C} / \mathrm{Fe}]$} & $\Delta[\mathrm{C} / \mathrm{Fe}]$ \\
\hline SDSS J012512+070319 & 77 & 6312 & 34 & 5.0 & 0.07 & -3.3 & 0.1 & 1.2 & 0.6 \\
SDSS J015131+1639441 & 62 & 6036 & 26 & 5.0 & 0.07 & -3.8 & 0.08 & 1.1 & 0.5 \\
SDSS J041800+062308 & 73 & 6247 & 35 & 5.0 & 0.08 & -3.4 & 0.1 & 0.7 & 0.7 \\
SDSS J094708+461010 & 80 & 5858 & 19 & 5.0 & 0.07 & -4.1 & 0.06 & 1.0 & 0.3 \\
SDSS J173403+644632 & 60 & 6183 & 35 & 5.0 & 0.09 & -4.3 & 0.11 & 3.1 & 0.1 \\
\hline G64-12 & 132 & 6393 & 29 & 4.8 & 0.07 & -3.2 & 0.1 & 1.0 & 0.4 \\
\hline
\end{tabular}

Notes. $\Delta$ are the internal uncertainties of parameters derived with FERRE.

References. (1) Aguado et al. (2017).

$\left(T_{\text {eff }}=6393 \mathrm{~K}, \log g=4.8,[\mathrm{Fe} / \mathrm{H}]=-3.2\right)$ and the literature (Aoki et al. 2006; Placco et al. 2016) $\left(T_{\text {eff }}=6390 \mathrm{~K}\right.$, $\log g=4.38,[\mathrm{Fe} / \mathrm{H}]=-3.2)$, together with the quality of the fit, suggest that this is a highly reliable method of confirming extremely metal-poor stars. Further details on FERRE tests are given by Allende Prieto et al. (2014), Aguado et al. (2017).

FERRE offers multiple options for deriving internal uncertainties. For our program we chose the estimates obtained from Monte Carlo experiments after injecting noise into the observations and the error treatment is the same as that included in Aguado et al. (2017). We derived the error bars by adding in quadrature the internal uncertainties showed in Table 3 with other sources of error: $\Delta \mathrm{T}_{\mathrm{eff}}=70 \mathrm{~K}, \Delta \log g=0.5, \Delta[\mathrm{Fe} / \mathrm{H}]=$ 0.1 dex and $\Delta[\mathrm{C} / \mathrm{Fe}]=0.2$ dex. The spectral range in common between the ISIS and the OSIRIS spectra is about $820 \AA$ wider, with the $\mathrm{H}_{\beta}$ line missing in the latter case. We examine the results for each object in Sect. 4.2.

\subsection{Testing the methodology with J1019+1729}

Caffau et al. (2011, 2012) recognized J1019+1729, a dwarf star at a metallicity of about $[\mathrm{Fe} / \mathrm{H}] \sim-5$, from SDSS/SEGUE spectroscopic data. The low carbon and oxygen abundances of this star challenge the picture of star formation that suggests no low-mass stars were formed (Bromm \& Loeb 2003). To test or methodology, we analyzed the X-shooter spectrum of this star $\left(R=7900\right.$ in the UVB arm). FERRE derives $T_{\text {eff }}=5834 \mathrm{~K}$, $\log g=5.0,[\mathrm{C} / \mathrm{Fe}]=-0.47$, and $[\mathrm{Fe} / \mathrm{H}]=-4.55$. The effective temperature derived by the authors $(5811 \mathrm{~K})$ is perfectly recovered by the code and is in agreement with our analysis of the ISIS spectrum of this target presented in Aguado et al. (2017). In addition, FERRE is able to identify J1029+1729 as a dwarf star. Since it is not visible, we are able to place an upper limit $[\mathrm{C} / \mathrm{Fe}]<+0.7$. However, our derived metallicity is higher than that determined by Caffau et al. (2012), due to the ISM contribution to the $\mathrm{Ca}$ II $\mathrm{H}$ and $\mathrm{K}$ lines. The metallicity is compatible with our analysis in Aguado et al. (2017) with ISIS and UVESsmoothed data. Furthermore, we ran the FERRE code only in the spectral region where the strongest Fe I lines are (3810$3865 \AA$ ) and assuming the previously derived $T_{\text {eff }}$ and $\log g=5.0$ over the entire $\mathrm{X}$-shooter spectrum, and we were able to recover $[\mathrm{Fe} / \mathrm{H}]=-4.75$ (see Fig. 1), which is in agreement with the 1D-LTE Fe I abundance derived by the authors in the original paper from higher resolution UVES spectroscopy. Finally, we smoothed the X-shooter spectrum of J1019+1729 to the OSIRIS resolution $(R=2500)$ and, again, we ran FERRE and arrived at the values $[\mathrm{Fe} / \mathrm{H}]=-4.5$ and $T_{\text {eff }}=5826 K$. This exercise

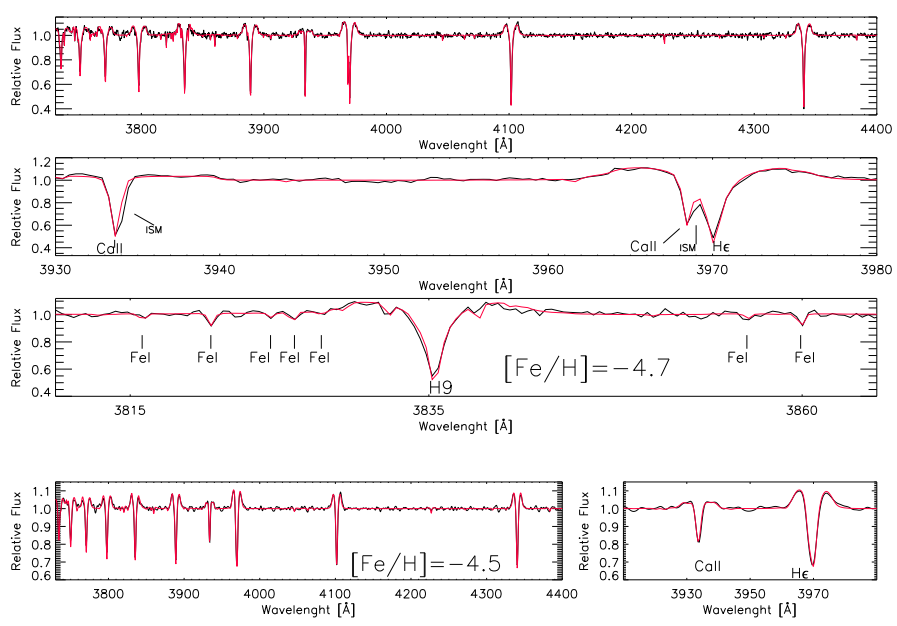

Fig. 1. VLT/X-shooter spectrum $(R=7900)$ of $\mathrm{J} 1019+1729$ (black line) and the best fit derived by FERRE (red line). From top to bottom: the entire spectrum (3700-4400 $)$ ). The range of the Ca II lines. The H9 spectral region with several Fe I lines assuming $T_{\text {eff }}=5834 \mathrm{~K}, \log g=$ 5.0 from our previous analysis over the entire spectra. The derived iron abundance is displayed. Finally the X-shooter spectrum smoothed to the OSIRIS resolution $(R=2500)$ and re-analyzed by FERRE: left panel shows the entire spectrum together with the derived metallicity while in the right panel a detail of the Ca II lines is depicted.

demonstrates that our methodology is robust and works even at higher resolution $(R=7900)$. We are able to recover the same metallicity and effective temperature from the analysis of SDSS, ISIS, and UVES-smoothed data (Aguado et al. 2017), and from $\mathrm{X}$-shooter and X-shooter-smoothed spectra.

\subsection{Discussion of the new set of faint metal-poor stars}

Figure 2 (upper panel) shows the spectrum of G64-12 (black solid line) and its best fit derived with FERRE. All spectra analyzed in this work are plotted below.

\subsubsection{J012512+070319}

The quality of the BOSS spectrum of this target is the highest in the sample. The initially derived set of parameters appears to be reliable; the much higher S/N OSIRIS spectra lead to an effective temperature that differs by only $140 \mathrm{~K}$. The slightly lower temperature we find decreases the derived metallicity by $0.1 \mathrm{dex}$. The final values for the parameters and metallicities of this object are $T_{\text {eff }}=6312 \pm 78 \mathrm{~K}, \log g=5.0 \pm 0.5$, 


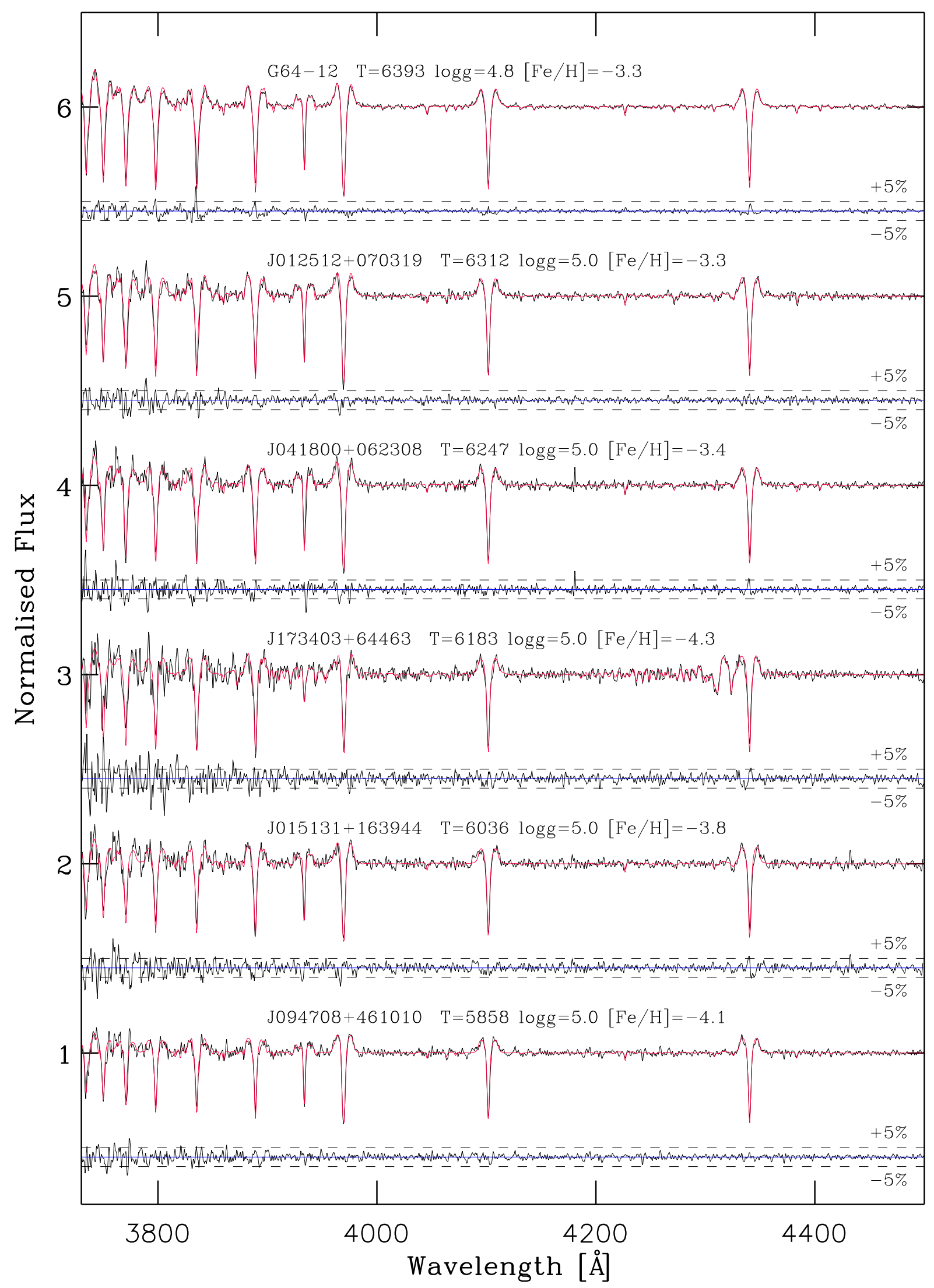

Fig. 2. GTC/OSIRIS spectra (3700-4500 A) our stellar sample (black line) and the best fit calculated with FERRE (red line). Under each of the spectra we also depict the residuals (difference between the observed spectrum and the best fit) together with the $+5 \%$ and $-5 \%$ reference lines. On top of each spectrum the main stellar parameters are displayed. 
D. S. Aguado et al.: New ultra metal-poor stars observed with GTC
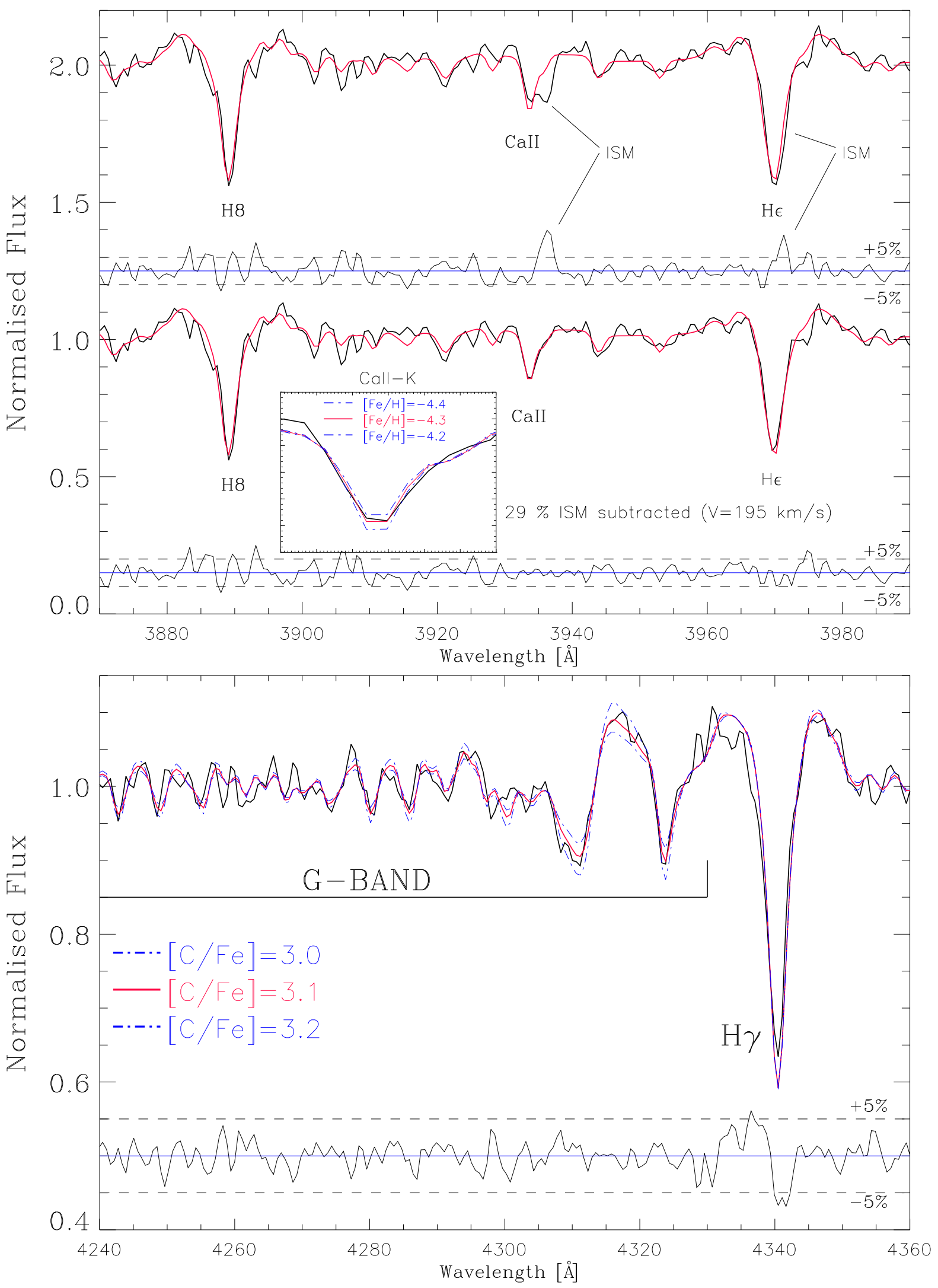

Fig. 3. Upper panel: spectral range in the vicinity of the Ca II H-K lines for the OSIRIS spectrum of the star J134157+513534 (black solid line) with the best fit (red line) before ISM subtraction (top panel) and after ISM subtraction (bottom panel). Below the spectra we display the residuals together with the $+5 \%$ and $-5 \%$ reference lines. A detail of the Ca II K line after ISM subtraction with an upper and lower metallicity limit (dashed blue line) is plotted. Lower panel: spectral range of the $G$-band of the OSIRIS spectrum of J134157+513534 (black solid line) with the synthetic spectra for the best fit (red line), an upper and lower limit of carbon abundances (dashed blue line). 
and $[\mathrm{Fe} / \mathrm{H}]=-3.3 \pm 0.2$. The derived carbon abundance is $[\mathrm{C} / \mathrm{Fe}]=1.2 \pm 0.6$.

\subsubsection{J015131+163944}

This object was observed with ISIS on the WHT and analyzed by Aguado et al. (2017). The set of derived parameters by these authors is $T_{\text {eff }}=6025 \pm 77 \mathrm{~K}, \log g=4.8 \pm 0.6,[\mathrm{Fe} / \mathrm{H}]=-3.8 \pm$ 0.2 and $[\mathrm{C} / \mathrm{Fe}]=1.3 \pm 0.4$, from a spectrum with very similar resolving power $(R \sim 2400)$ and slightly lower $\mathrm{S} / \mathrm{N}$ than the OSIRIS spectrum presented here. The values given in Table 3 are essentially the same: $T_{\text {eff }}=6036 \pm 75 \mathrm{~K}, \log g=5.0 \pm 0.5$, $[\mathrm{Fe} / \mathrm{H}]=-3.8 \pm 0.2$ and $[\mathrm{C} / \mathrm{Fe}]=1.1 \pm 0.5$.

\subsubsection{J041800+062308}

The SEGUE spectrum of this object has a fairly poor quality, and, as a result, the derived effective temperature, $T_{\text {eff }}=5579 \mathrm{~K}$, is not very reliable. The set of parameters from the OSIRIS analysis $\left(T_{\text {eff }}=6247 \pm 78 \mathrm{~K}, \log g=5.0 \pm 0.5,[\mathrm{Fe} / \mathrm{H}]=-3.4 \pm 0.2\right)$ are more robust, while the $G$-band is close to the detection limit $([\mathrm{C} / \mathrm{Fe}]=0.7 \pm 0.7)$.

\subsubsection{J094708+461010}

The $\mathrm{S} / \mathrm{N}$ of this BOSS spectrum is the lowest in the sample, and for this reason we consider the derived atmospheric parameters in Table 1 unreliable. However, both the effective temperature and the gravity derived from the OSIRIS spectrum are compatible with those from the BOSS observations. Accepting the FERRE derived values, we arrive at $T_{\text {eff }}=5858 \pm 73 \mathrm{~K}$, $\log g=5.0 \pm 0.5,[\mathrm{Fe} / \mathrm{H}]=-4.1 \pm 0.2$, and $[\mathrm{C} / \mathrm{Fe}]=1.0 \pm$ 0.4 . Interestingly enough, the Yoon-Beers diagram described in Yoon et al. (2016) points out that there are two different "families" of CEMP-no stars, which they designate as Group II and Group III. J094708+461010 is a Group II with $[\mathrm{Fe} / \mathrm{H}]=-4.1$ and $\mathrm{A}(\mathrm{C})=5.3$.

\subsubsection{J173403+644632}

The spectrum of this target shows the clearest and unresolved calcium ISM contribution, and the strongest $G$-band absorption, making this faint object very interesting candidate for a detailed study. Since the Ca II H-K spectral region (the one containing more information) is affected by $\mathrm{CH}$ lines, we perform the analysis with FERRE as follows:

- We derived simultaneously the effective temperature $\left(T_{\text {eff }}=\right.$ $6183 \mathrm{~K}$ (see Fig. 3, upper panel), the carbon abundance $([\mathrm{C} / \mathrm{Fe}]=3.1$ : see Fig. 3 , lower panel $)$, and a surface gravity of $\log g=5.0$.

- A Gaussian profile for the calcium ISM contribution is adopted. A grid of varying absorption coefficients $(\epsilon)$ from $5 \%$ to $30 \%$ in step sizes of $1 \%$ and relative velocities from $180 \mathrm{~km} \mathrm{~s}^{-1}$ to $220 \mathrm{~km} \mathrm{~s}^{-1}$ in steps of $5 \mathrm{~km} \mathrm{~s}^{-1}$ were subtracted, and thus the resulting spectrum was reanalyzed with FERRE.

- The minimum $\chi^{2}$ is obtained for the point $\epsilon=0.29$ and $V=195 \mathrm{~km} \mathrm{~s}^{-1}$ which reproduces best both the Ca II and the $\mathrm{H}_{\gamma}$ lines (see Fig. 3, upper panel).

Thus, the final set of parameters are $T_{\mathrm{eff}}=6183 \pm 78 \mathrm{~K}$, $\log g=5.0 \pm 0.5,[\mathrm{Fe} / \mathrm{H}]=-4.3 \pm 0.2$, and $[\mathrm{C} / \mathrm{Fe}]=$ 3.1 \pm 0.2. Again, regarding the diagram mentioned in Sect. 4.2.4, $\mathrm{J} 173403+644632$ with $[\mathrm{Fe} / \mathrm{H}]=-4.3$ and $\mathrm{A}(\mathrm{C})=7.2$ would clearly be in the Group III This target is the only ultra metalpoor star known at $V>19$. Finally, the fact that the derived

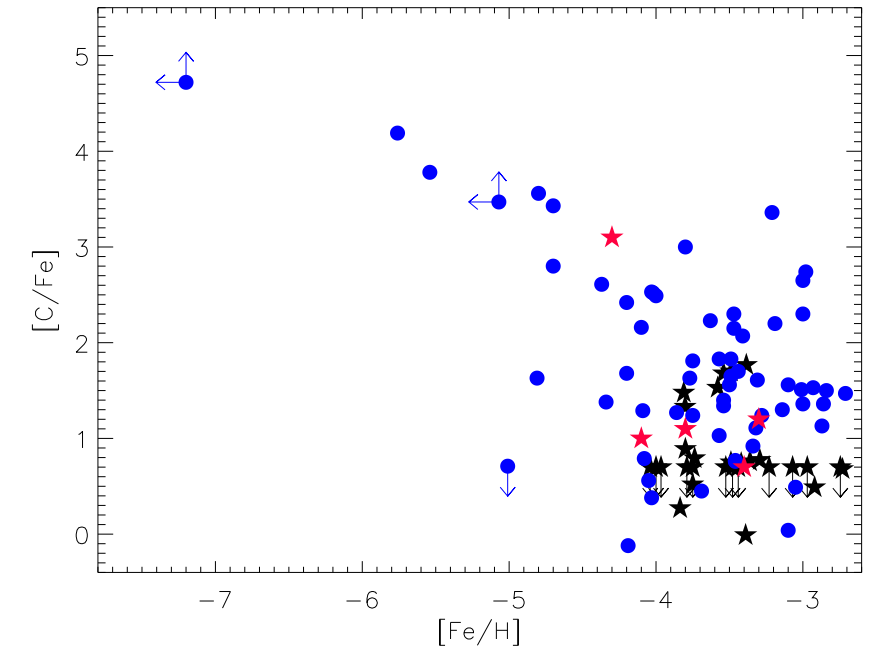

Fig. 4. Carbon over iron versus metallicities of stars presented in this work (red filled stars) and those from Aguado et al. (2017; black filled stars) analyzed with the improved methodology, together with stars from the literature (Sivarani et al. 2006; Yong et al. 2013; Frebel et al. 2005, 2006; Caffau et al. 2014; Allende Prieto et al. 2015) represented by blue filled circles.

gravities are always on the limit of the grid is compatible with the relatively high error bars adopted. However, the influence on the derived metallicity and carbon abundance is small and already considered in error bars through other sources of error (Aguado et al. 2017).

\section{Conclusions}

The multiple techniques use to identify and confirm metalpoor stars have been summarized by Beers \& Christlieb (2005), Frebel \& Norris (2015), and references therein, and include high proper-motion surveys, Schmidt objective-prism surveys, photometric surveys, and spectroscopic surveys. The most iron-poor star known, SM 0313-6708 with $[\mathrm{Fe} / \mathrm{H}]<-7.3$, was discovered from a photometric survey, the Skymapper Southern Sky Survey (Keller et al. 2014). The most metal-poor star known, $\mathrm{J} 1029+1729$, was in turn recognized in the SDSS spectroscopic survey by Caffau et al. (2012). Obviously, the observational efforts and resources employed demand less telescope timewhen using photometry to identify metal-poor stars (Starkenburg et al. 2017), but the only path to confirm and derive reliable metallicities involves spectroscopic techniques.

Usually a low-resolution spectroscopic analysis is required to securely identify metal-poor candidates, and the subsequent and significantly more expensive high-resolution analysis confirms the metallicity and provides multiple chemical abundances. Avoiding false positives (incorrect identifications) optimizes the invested time in 6-10 $\mathrm{m}$ class telescopes. In this paper we use an intermediate step (see Beers et al. 1985) to increase this ratio. Using medium-resolution spectroscopy we are able to derive reliable metallicities and sometimes carbon abundances, even in the $[\mathrm{Fe} / \mathrm{H}]<-4.0$ regime.

We have identified a number of very faint metal-poor stars, and offer to the community a sizable sample of faint ultra metalpoor stars to observe with the next generation of 30-40 m telescopes. Figure 4 shows the carbon abundances derived from OSIRIS spectra for the targets studied in this work, together with those from Aguado et al. (2017), and other CEMP stars from the literature. The chance to carry out a follow-up program for observing stars with $V>19$ significantly improves our success ratio of identifying ultra metal-poor stars. The more 
carbon-enhanced metal-poor stars are those that are below the $[\mathrm{Fe} / \mathrm{H}]=-4.0$ regime.

In addition, we demonstrate the advantages of our methodology when the calcium ISM contribution is not resolved at medium resolution which is the vast majority. However, the most interesting star of this work, J173403+644632, is an excellent candidate to observe at higher resolution spectroscopy and to derive its chemical composition in detail, giving important constraints to the early Universe. Finally, we have observed and analyzed, with highly reliable results, well-known metal-poor stars (G64-12 and J1029+1729), together with those presented in Aguado et al. (2017), and this allows us to trust our methodology when looking for ultra metal-poor stars.

Acknowledgements. D.A. acknowledges the Spanish Ministry of Economy and Competitiveness (MINECO) for the financial support received in the form of a Severo-Ochoa Ph.D. fellowship, within the Severo-Ochoa International Ph.D. Program. D.A., C.A.P., J.I.G.H., and R.R. also acknowledge the Spanish ministry project MINECO AYA2014-56359-P. J.I.G.H. acknowledges financial support from the Spanish Ministry of Economy and Competitiveness (MINECO) under the 2013 Ramón y Cajal program MINECO RYC-2013-14875. This paper is based on observations made with the Gran Telescopio de Canarias, operated by the GRANTECAN team at the Observatorio del Roque de los Muchachos, La Palma, Spain, of the Instituto de Astrofísica de Canarias. We thank the GRANTECAN staff members in general and Antonio Cabrera Lavers and Daniel Reverte Payá in particular for their efficiency during the program preparation.

\section{References}

Aguado, D. S., Allende Prieto, C., González Hernández, J. I., et al. 2016, A\&A, 593, A10

Aguado, D. S., González Hernández, J. I., Allende Prieto, C., \& Rebolo, R. 2017, A\&A, in press, DOI: 10.1051/0004-6361/201730654
Ahn, C. P., Alexandroff, R., Allende Prieto, C., et al. 2012, ApJS, 203, 21 Alam, S., Albareti, F. D., Allende Prieto, C., et al. 2015, ApJS, 219, 12 Allende Prieto, C., Beers, T. C., Wilhelm, R., et al. 2006, ApJ, 636, 804 Allende Prieto, C., Fernández-Alvar, E., Schlesinger, K. J., et al. 2014, A\&A, 568, A7

Allende Prieto, C., Fernández-Alvar, E., Aguado, D. S., et al. 2015, A\&A, 579, A98

Allende Prieto, C., Kawata, D., \& Cropper, M. 2016, A\&A, 596, A98

Aoki, W., Frebel, A., Christlieb, N., et al. 2006, ApJ, 639, 897

Beers, T. C., \& Christlieb, N. 2005, Highlights of Astronomy, 13, 579

Beers, T. C., Preston, G. W., \& Shectman, S. A. 1985, AJ, 90, 2089

Bonifacio, P., Caffau, E., Spite, M., et al. 2015, A\&A, 579, A28

Bromm, V., \& Loeb, A. 2003, Nature, 425, 812

Caffau, E., Bonifacio, P., François, P., et al. 2011, Nature, 477, 67

Caffau, E., Bonifacio, P., François, P., et al. 2012, A\&A, 542, A51

Caffau, E., Sbordone, L., Bonifacio, P., et al. 2014, Mem. Soc. Astron. Italiana, 85,222

Dawson, K. S., Schlegel, D. J., Ahn, C. P., et al. 2013, AJ, 145, 10

Eisenstein, D. J., Weinberg, D. H., Agol, E., et al. 2011, AJ, 142, 72

Frebel, A., \& Norris, J. E. 2015, ARA\&A, 53, 631

Frebel, A., Aoki, W., Christlieb, N., et al. 2005, Nature, 434, 871

Frebel, A., Christlieb, N., Norris, J. E., et al. 2006, ApJ, 652, 1585

Keller, S. C., Bessell, M. S., Frebel, A., et al. 2014, Nature, 506, 463

Koesterke, L., Allende Prieto, C., \& Lambert, D. L. 2008, ApJ, 680, 764

Mészáros, S., Allende Prieto, C., Edvardsson, B., et al. 2012, AJ, 144, 120

Placco, V. M., Frebel, A., Lee, Y. S., et al. 2015, ApJ, 809, 136

Placco, V. M., Beers, T. C., Reggiani, H., \& Meléndez, J. 2016, ApJ, 829, L24

Sivarani, T., Beers, T. C., Bonifacio, P., et al. 2006, A\&A, 459, 125

Smee, S. A., Gunn, J. E., Uomoto, A., et al. 2013, AJ, 146, 32

Starkenburg, E., Martin, N., Youakim, K., et al. 2017, MNRAS, submitted [arXiv: 1705.01113]

Tody, D. 1993, in Astronomical Data Analysis Software and Systems II, eds. R. J. Hanisch, R. J. V. Brissenden, \& J. Barnes, ASP Conf. Ser., 52, 173

Yanny, B., Rockosi, C., Newberg, H. J., et al. 2009, AJ, 137, 4377

Yong, D., Norris, J. E., Bessell, M. S., et al. 2013, ApJ, 762, 26

Yoon, J., Beers, T. C., Placco, V. M., et al. 2016, ApJ, 833, 20

York, D. G., Adelman, J., Anderson, Jr., J. E., et al. 2000, AJ, 120, 1579 